\title{
CAPACIDADE DE INOVAÇÃO NAS EMPRESAS INCUBADAS
}

\author{
E. A. NOBRE ${ }^{1 *}$, R. P. S. COSTA ${ }^{1}$, W. A. EL-AOUAR ${ }^{2}$, L. K. S. BARRETO ${ }^{2}$ e L. A. SOUZA ${ }^{2}$ \\ ${ }^{1}$ Instituto Federal de Educação, Ciência e Tecnologia do Rio Grande do Norte \\ ${ }^{2}$ Universidade Potiguar \\ elizomar.nobre@ifrn.edu.br*
}

Artigo submetido em novembro/2015 e aceito em abril/2016

DOI: 10.15628/holos.2016.3696

\section{RESUMO}

O estudo apresentado neste artigo tem como objetivo compreender a capacidade de inovação das empresas incubadas na perspectiva da Incubadora Tecnológica Natal Central (ITNC). Para atingir esse objetivo foram realizadas entrevistas com a equipe da ITNC, no intuito de avaliar a capacidade de inovação das empresas incubadas. A partir dos resultados dessas empresas, pode-se identificar, do ponto de vista da ITNC, a presença dos fatores que constituem a capacidade de inovação, sendo a intenção estratégica de inovar e a gestão de projetos percebidos de maneira mais sutil, a organicidade da estrutura organizacional e a gestão estratégica da tecnologia notada como predominantes no dia a dia dessas empresas.

PALAVRAS-CHAVE: Capacidade de Inovação, Empresas Incubadas, Incubadoras, Inovação.

\section{INNOVATION CAPACITY IN INCUBATED COMPANIES}

\begin{abstract}
This study is this articles tries to understand the innovation capacity of incubator companies in the perspectives of Incubadora Tecnológica Natal Central (ITNC). To reach this objective interviews were accomplished with the ITNC team to evaluate the innovation capacity of the incubator companies. In the results it is possible to identity, from the ITCN point of
\end{abstract}

view, the presence of aspects that show the capacity of innovation, where the strategic intention to innovate and the projects administration were slightly detected, as well as the arrangement of the strategic administration of technology were predominant in the routine of the companies.

KEYWORDS: Innovation capacity, Business incubators, Incubator, innovation. 


\section{INTRODUÇÃO}

Empreendedorismo é o processo pelo qual o conhecimento criado por uma organização se torna comercial numa empresa. É o comportamento das pessoas definido como a capacidade de responder a um ambiente a fim de construir soluções que agregarão valor à sociedade. Segundo Paiva (2005), é a habilidade de transformar ideias em ações.

As incubadoras de empresas exercem um papel fundamental na relação entre empreendedorismo, inovação e mercado. Sua infraestrutura funciona como mecanismo-chave na formação de novas organizações e no aprimoramento de empresas e produtos, oferecendo o espaço necessário, a consultoria e a capacitação. Nos últimos anos, essa estrutura tem despertado interesse nos diversos seguimentos políticos, agências de desenvolvimento, empreendedores, instituições de pesquisa e acadêmicas (DE FELICE, 2014).

A concepção de incubadoras de empresas ficou conhecida na década de 1950, na região hoje conhecida como Vale do Silício, na Califórnia, a partir das iniciativas da Universidade de Stanford, com o objetivo de transferir tecnologia desenvolvida na universidade às empresas e à criação de novos empreendimentos. O êxito foi de tal forma que estimulou a reprodução de iniciativas semelhantes em outras localidades como Japão, China, Índia, México, Argentina, Turquia, Polônia, Portugal entre outras localidades. Internacionalmente, as incubadoras têm provado ser um modelo extremamente bem-sucedido, pois estimula o desenvolvimento econômico e o crescimento de emprego e renda.

No Brasil, o papel das políticas públicas no investimento das incubadoras tem sido primordial para o desenvolvimento destas como mecanismos de aceleração do crescimento de empresas inovadoras. O Ministério de Ciência e Tecnologia (MCTI) definiu o Plano de Ação para Ciência e Tecnologia e Inovação entre 2007 a 2010 (PACTI), ao qual promove a inovação tecnológica do país, proporcionando mecanismos, fomentados pelo governo federal, como indutores do processo de inovação tecnológica, como é o caso das incubadoras de empresas de base tecnológica. Para o MCTI, o processo de incubação é um dos mais eficazes mecanismos de formação de empresas.

No Brasil, esse trabalho com incubadoras teve início na década de 1980 , por inciativa do Conselho Nacional de Desenvolvimento Científico e Tecnológico (CNPq), com o propósito de fomentar a cultura empreendedora de pesquisa e desenvolvimento, bem como disseminar atividades inovadoras no setor produtivo.

No Rio Grande do Norte, existem ao todo 12 incubadoras (SEBRAE, 2015), sendo a primeira delas, a Incubadora Tecnológica Natal-Central (ITNC), fundada em 1998, na Escola Técnica Federal do Rio Grande do Norte (ETFERN), hoje Instituto Federal de Educação, Ciência e Tecnologia do Rio Grande do Norte (IFRN). Além da ITNC, o IFRN conta com as seguintes incubadoras: Incubadora de Cultura e Arte (ITCART), localizada no Campus Cidade Alta, em Natal; Incubadora Tecnológica e de Negócios do Mato Grande (ITEN), localizada no Campus João Câmara; Incubadora em Tecnologia Têxtil (ITEXTIL), localizada no Campus Caicó; Incubadora Tecnológica de Mossoró (ITMO), localizada no Campus Mossoró; e Incubadora Tecnológica em Inovação em Processamento de Alimentos e Serviços (ITIPAS), localizada no Campus Pau dos Ferros. Isso demonstra que o IFRN é a instituição com maior número de incubadoras implementadas no estado. 
A ITNC, objeto deste estudo, atua no fomento à criação e à implantação de novas empresas, levando suporte técnico aos futuros empreendedores, orientando na criação da empresa, transferindo tecnologia, estimulando a inovação e o empreendedorismo, permitindo, dessa forma, o desenvolvimento tecnológico de empresas nascentes ou já em funcionamento nos arranjos produtivos locais.

Para ser incubada a empresa precisa demonstrar o seu potencial interno na identificação de novas ideias, mercados e oportunidades, ou seja, a empresa ou a ideia de negócio tem que ser inovadora e ter perspectiva de mercado, requisitos básicos no processo de seleção, que envolvem um leque de competências e habilidades gerenciais, tecnológicas e mercadológicas.

A partir desse entendimento, o foco deste trabalho volta-se a compreender a capacidade de inovação das empresas incubadas na perspectiva da equipe da ITNC do IFRN, com o intuito de descrever os conceitos, tipologias e características da inovação e sua importância para as empresas; apresentar o ponto de vista da equipe da ITNC quanto à atuação das incubadas, suas potencialidades e/ou fragilidades, e, enfim, identificar os fatores determinantes e os fatores resultantes da capacidade de inovação das empresas abrigadas na ITNC.

Isto posto, o presente artigo estrutura-se a partir da introdução, seguida do referencial teórico, o qual abrange os conceitos, as tipologias e a classificação da inovação; caracteriza empresa empreendedora; descreve o que é e como funcionam as incubadoras de empresas, destacando o papel da Incubadora Tecnológica Natal-Central; e, apresenta ainda a definição da capacidade de inovação. Em seguida, são apresentados os achados obtidos por meio das entrevistas realizadas com a equipe gestora da ITNC. E, finalmente, são tecidas as considerações finais sobre os resultados encontrados na pesquisa, suas contribuições e a indicação de novos pontos a serem investigados sobre o tema abordado.

\section{REFERENCIAL TEÓRICO}

\subsection{Inovação}

A palavra inovação traz em si a ideia de novidade, algo novo ou modificado. Segundo o Manual de Oslo (2005), criado pela Organização para Cooperação e o Desenvolvimento Econômico (OCDE), inovação é a implementação de um novo ou o melhoramento de um produto, processo ou método. Para Quinello e Nicoletti (2009), além da introdução de novos bens e serviços, também se qualificam como inovação: uma nova estrutura organizacional, um novo modo de pensar e aprender, uma nova forma de agir ou um novo comportamento.

Com essa mesma compreensão, Hashimoto (2006) julga inovação qualquer pequena mudança que provoca uma transformação, numa perspectiva de desenvolvimento do pensamento de ruptura e uma quebra de paradigmas que permitam entender e visualizar o tradicional como passível de mudanças positivas e melhorias.

O tema inovação começou a ser destaque no início do século $X X$, época de intensa industrialização, quando o economista austríaco e professor da Universidade de Harvard, Joseph Alois Schumpeter, estabeleceu uma correlação entre desenvolvimento econômico e inovação. Schumpeter defendia que a introdução de novos arranjos produtivos ou mudanças nas funções de produção levavam à reorganização da atividade econômica, impulsionando o crescimento da economia (QUINELLO; NICOLETTI, 2009). 
Nesse sentido, Schumpeter (1997) define inovação com bases em cinco aspectos principais: 1) introdução de um novo bem ou de uma nova qualidade de um bem; 2 ) introdução de um novo método de produção; 3) abertura de um novo mercado; 4) conquista de uma nova fonte de oferta de matérias-primas ou de bens semimanufaturados; e 5) estabelecimento de uma nova organização comercial.

Schumpeter (1997) descreve ainda uma relação entre inovação, criação de novos mercados e a ação de empreender ao afirmar que o produtor inicia a mudança econômica a partir da inserção de algo novo, educando a população a desejar novas coisas, ou coisas que diferem de alguma forma daquelas habitualmente consumidas, substituindo antigos hábitos de consumo ou produtos por novos. De forma geral, ocorre um processo sistemático pelo qual cria-se um produto (bem ou serviço), em seguida, estimula-se a demanda e o consumo, provocando, consequentemente, uma variação positiva no desenvolvimento econômico.

No entanto, vale lembrar que o desenvolvimento econômico vai além dos progressos ligados à produção de bens e à acumulação de capital. Está associado também aos aspectos relacionados ao bem-estar social. Seria, então, o equilíbrio entre as condições de consumo e qualidade de vida da população (BASSAN, 2014).

Dessa maneira, entende-se que as empresas são fundamentais para o progresso da sociedade, uma vez que exploram oportunidades de negócios, desenvolvem novos produtos ou processos, implementam-nos no mercado e assim criam, aprimoram e introduzem a inovação.

\subsubsection{Classificação da Inovação}

Existem no conceito de inovar diferentes formas de inovação, classificadas de acordo com sua aplicação. A OCDE (2005) classifica quatro tipos diferentes de inovação em seu Manual de Oslo: 1) inovação de produto; 2) inovação de processo; 3) inovação organizacional; e 4) inovação em marketing.

Quadro 1: Tipos de Inovação

\begin{tabular}{|c|c|c|}
\hline Tipo & Objeto & Exemplos \\
\hline Inovação de produto & $\begin{array}{l}\text { Introdução de um bem ou serviço novo ou } \\
\text { significativamente melhorado no que tange a } \\
\text { suas características ou usos previstos. }\end{array}$ & $\begin{array}{l}\text { Melhoramentos significativos em } \\
\text { especificações técnicas, componentes e } \\
\text { materiais, softwares incorporados, } \\
\text { facilidade de uso ou outras características } \\
\text { funcionais. }\end{array}$ \\
\hline Inovação de processo & $\begin{array}{l}\text { Inserção de um método de produção ou } \\
\text { distribuição novo ou significativamente } \\
\text { melhorado. }\end{array}$ & $\begin{array}{l}\text { Mudanças significativas em técnicas, } \\
\text { equipamentos e/ou softwares. }\end{array}$ \\
\hline Inovação de marketing & $\begin{array}{l}\text { Implementação de um novo método de } \\
\text { marketing com mudanças significativas na } \\
\text { concepção do produto ou em sua } \\
\text { embalagem, no posicionamento do produto, } \\
\text { em sua promoção ou na fixação de preços. }\end{array}$ & $\begin{array}{l}\text { Mudanças substanciais no design do } \\
\text { produto, variedade de produtos, novos } \\
\text { canais de vendas, constituição de um novo } \\
\text { conceito de marketing. }\end{array}$ \\
\hline Inovação organizacional & $\begin{array}{l}\text { Adoção de um novo método organizacional } \\
\text { nas práticas de negócios da empresa, na } \\
\text { organização do seu local de trabalho ou em } \\
\text { suas relações externas. }\end{array}$ & $\begin{array}{l}\text { Novos métodos para a organização de } \\
\text { rotinas e procedimentos para a condução } \\
\text { do trabalho. }\end{array}$ \\
\hline
\end{tabular}

Fonte: Manual de Oslo (OCDE, 2005).

Muitas inovações podem ter características que aparecem em mais de um tipo de inovação, como uma empresa que lança um novo produto e para isso, adota um novo método organizacional 
ou um novo processo. Isso demonstra que, em alguns casos, os variados tipos de inovação também se complementam. No entanto, é importante distingui-los para melhor entendê-los e explorá-los.

Por sua vez, os pesquisadores Henderson e Clark (1990) caracterizam os diferentes tipos de inovação consoante impacto que produzem na empresa, sob duas dimensões: horizontal e vertical. A dimensão horizontal compreende o impacto de uma inovação em componentes, classificando em: 1) inovação incremental; 2) inovação modular; 3) inovação arquitetural; e 4) inovação radical. Enquanto a dimensão vertical capta seu impacto sobre as ligações entre os componentes da empresa, dividindo em mutável e imutável.

A inovação incremental refere-se a poucas modificações, introduzidas apenas para aperfeiçoar e ampliar as competências existentes, comuns em empresas estabelecidas. Já a inovação radical refere-se a grandes mudanças, demandam habilidades, abordagens e conceitos diferentes das práticas habituais da organização (QUINELLO; NICOLETTI, 2009).

A inovação modular é basicamente a substituição de um componente, ou seja, muda o conceito de um produto sem alterar sua arquitetura. Enquanto a inovação arquitetural é a reconfiguração de um sistema estabelecido unindo os componentes de uma nova maneira (HENDERSON; CLARK, 1990).

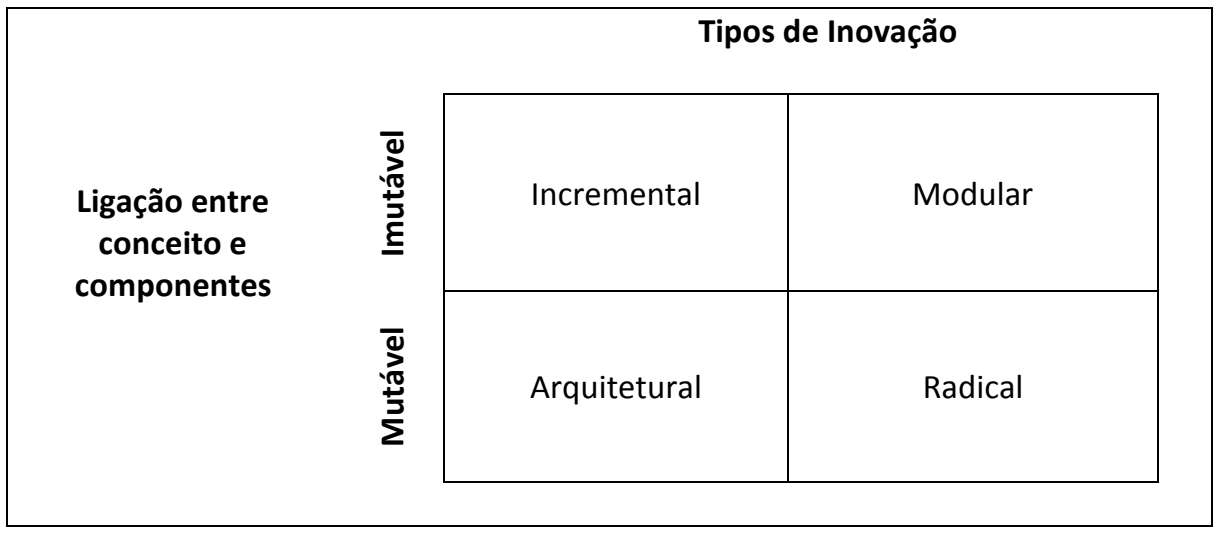

Figura 1- Tipos de inovação $x$ impacto na empresa Fonte: Henderson e Clark (1990).

A inovação também pode ser classificada considerando a capacidade inovadora de forma empreendedora, por meio da matriz de oportunidades proposta por Muzyka e Churchill (1995). A matriz é composta por duas dimensões: a fonte de inovação e a natureza da oportunidade. As fontes são: 1) internas: recursos existentes dentro da empresa; 2 ) externas: recursos externos à empresa; e 3) redes: conjuntos de fontes que misturam fontes e internas e externas (HASHIMOTO, 2006).

$\mathrm{Na}$ matriz, a natureza da oportunidade classifica a inovação como: 1) incremental: agregação de valor ao produto já existente por meio de serviços agregados e/ou novas funcionalidades; 2) evolucionária: provoca grandes mudanças, como lançamentos de novos modelos; e 3) descontínua ou radical: criação de um produto totalmente novo, sem qualquer semelhança com o atual, estabelecendo novos conceitos e/ou nova estruturação (HASHIMOTO, 2006). 


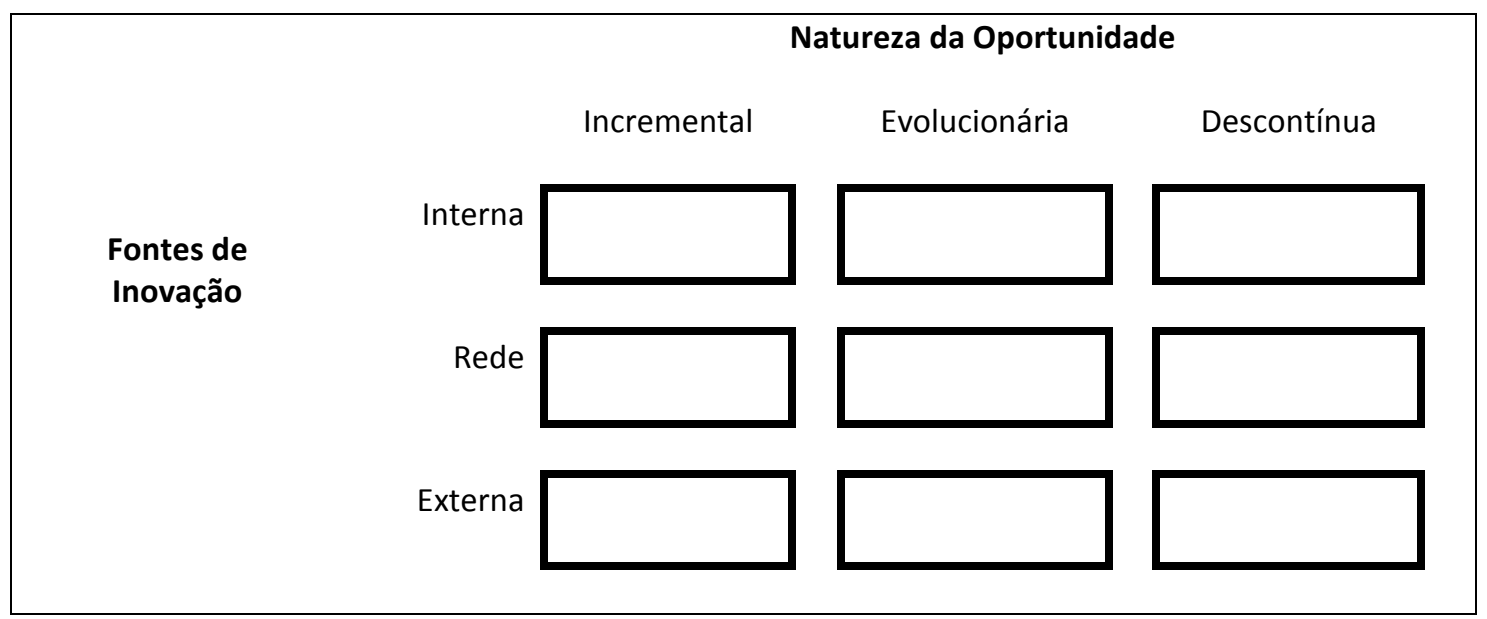

Figura 2- Inovação $x$ natureza da oportunidade

Fonte: Muzyka e Churchill apud Hashimoto (2006).

Observa-se, então, que existem diferentes formas de inovar. De pequenas a grandes modificações, que incrementam valorizando o potencial existente ou revolucionam, instituindo novos bens ou serviços, criados para otimizar e melhorar a atuação da empresa. Com isso, se faz necessário classificar a inovação, a fim de compreender seus aspectos e avaliar as mudanças que trarão para empresa, observando sua estrutura e a capacidade de inovação.

\subsubsection{Empresa inovadora}

A premissa básica de uma empresa inovadora é ter implementado ao menos um tipo de inovação ou combinações de inovações. Para inovar as empresas empreendem atividades de dois tipos: pesquisa e desenvolvimento (P\&D) e outras atividades que envolvem a aquisição de bens, serviços e conhecimentos externos. As atividades inovadoras dependem da variedade e da estrutura de suas interações com as fontes de informação, conhecimentos, tecnologias, práticas, recursos humanos e financeiros. Todas as atividades de inovação têm como objetivo final a melhoria do desempenho da empresa (OCDE, 2005).

As inovações nas empresas referem-se a mudanças planejadas na sua atuação com o intuito de melhorar seu desempenho e alcançar vantagem competitiva. A partir dessa percepção, entre os fatores motivadores para inovação, podemos destacar: redirecionar a produção, reduzir custos, melhorar a qualidade, aumentar a receita, melhorar o atendimento ao cliente e aumentar a lucratividade (QUINELLO; NICOLETTI, 2009).

Uma empresa inovadora adota uma série de práticas de gestão que melhoram a utilização de recursos e usam a inovação como estratégia para posicionar-se de forma diferenciada no mercado, gerando uma vantagem competitiva sustentável. Para tanto é necessário considerar seis práticas fundamentais: 1 ) entender o que deseja alcançar, dentro de uma perspectiva tangível de crescimento; 2) atinar um ponto de vista estruturado sobre o futuro; 3 ) definir os campos de pesquisa de inovação, construindo sobre as tendências do ambiente operacional atual e do mundo em geral; 4) gerenciar para resultado desejado do cliente; 5) conhecer suas próprias competências e investir em conformidade; e 6) desenhar um roadmap, como forma de mapear seus processos de desenvolvimento de inovação (ENGEL et al, 2015).

As empresas inovadoras admitem novos modelos para organizar e gerenciar suas atividades, bem como lançam novos produtos, modificam processos, exploram novos nichos de 
mercado, mudam regras, não se adaptam ao convencional, geram novas demandas, e assim, criam uma vantagem competitiva frente às demais empresas.

Entretanto, são ainda necessários métodos de identificação do potencial de inovação das empresas, baseados nos tipos de inovações que elas empreendem e suas capacidades e atividades inovadoras (OCDE, 2005). Estudos apontam que a capacidade de inovação está associada às práticas e aos processos executados pela empresa e que tais elementos têm efeitos sobre o desempenho nos negócios (SAUNILA; UKKO, 2012).

\subsection{Incubadoras de empresas}

Considerando a literatura existente, o conceito de incubação de empresas refere-se a um processo concentrado em sustentar empresas no estágio inicial de sua atividade, oferecendo um ambiente configurado para protegê-las contra falhas e orientá-las em um caminho de crescimento, desenvolvimento e fortalecimento, preparando-as para sobreviver ao mercado (THEODORAKOPOULOS et al, 2014).

De acordo com a Associação de Parques Tecnológicos e Incubadoras de Empresas Anprotec (2002), incubadora pode ser definida sob três aspectos: a) promotora do desenvolvimento, geração e consolidação de micro e pequenas empresas; b) sistema que impulsiona a criação e o desenvolvimento de micro e pequenas empresas por meio da formação complementar do empreendedor em seus aspectos técnicos e gerenciais; e c) facilitador do processo de empresariamento e inovação tecnológica para micro e pequenas empresas.

Internacionalmente, as incubadoras têm provado ser um modelo extremamente bemsucedido, uma vez que estimula o desenvolvimento econômico, crescimento do emprego e renda. Estima-se que atualmente existem 7.000 incubadoras em todo o mundo. Entre estes, cerca de 1.800 estão nos Estados Unidos e 900 na Europa (AL-MUBARAKI; BUSLER, 2014).

No Brasil, as primeiras incubadoras surgiram na década de 1980, por diligência do Conselho Nacional de Desenvolvimento Científico e Tecnológico (CNPq), agência do Ministério da Ciência, Tecnologia e Inovação (MCTI), com o propósito de fomentar a cultura empreendedora de pesquisa e desenvolvimento (MORAIS, 1997).

Um estudo realizado em 2011 pela Anprotec e pelo MCTI aponta que o movimento das incubadoras brasileiras atingiu a maturidade, entrando numa fase de profissionalismo e de qualificação do processo de gestão. Atualmente, são 384 incubadoras em operação, que abrigam 2.640 empresas, gerando 16.394 postos de trabalho. Essas incubadoras também já graduaram 2.509 empreendimentos, que hoje faturam $R \$ 4,1$ bilhões e empregam 29.205 pessoas (ANPROTEC, 2012.)

O processo de incubação é mundialmente reconhecido como um dos mecanismos mais eficazes para lançar e desenvolver novos empreendimentos. No Brasil e no mundo as estatísticas revelam que a taxa de mortalidade de empresas que passam pelo processo de incubação é reduzida de $70 \%$ para $20 \%$ em comparação com as empresas iniciadas, normalmente, por conta e risco dos empreendedores (ARAúJO et al, 2013).

As incubadoras no Brasil são classificadas de acordo com os tipos de empreendimentos que abrigam. A saber: 1) incubadoras de base tecnológica; 2) incubadoras tradicionais; 3) incubadoras mistas; 4) incubadoras sociais; e 5) incubadoras de cooperativas. As incubadoras de base 
tecnológica alojam as empresas cujos produtos, processos ou serviços resultam de pesquisa científica, nos quais a tecnologia representa alto valor e agregado, enquanto as incubadoras tradicionais abrigam as empresas de diferentes setores da economia, que queiram agregar valor aos seus produtos, processos ou serviços, mas que utilizam tecnologia largamente difundida. As incubadoras mistas compreendem tanto empreendimentos de base tecnológica quanto de setores tradicionais da economia e as incubadoras sociais e de cooperativas abrigam os empreendimentos oriundos de projetos sociais e associativos. Além dessas, ainda existem incubadoras culturais, de serviços e agroindustriais (ANPROTEC, 2002).

De acordo com o Relatório Técnico da Anprotec (2012), finalizado em 2011, feito para levantar e atualizar as informações e os indicadores das incubadoras brasileiras, $40 \%$ das incubadoras no país são de base tecnológica, $18 \%$ são tradicionais, $18 \%$ mistas, $8 \%$ de serviços, $7 \%$ são sociais, $7 \%$ são agroindustriais e $2 \%$ são culturais.

As incubadoras são, geralmente, vinculadas às instituições de ensino públicas ou privadas, prefeituras, centros de pesquisa, empresas ou associações de classe que têm como objetivo incentivar a criação de novos empreendimentos, apoiar o desenvolvimento da capacidade de inovação de pequenas e médias empresas e disseminar a cultura do empreendedorismo, voltado ao conhecimento e à inovação. Essas organizações dispõem de espaço físico apara alojar as empresas; infraestrutura e serviços compartilhados como salas de reuniões, telefone, informática; assessoria técnica e empresarial; maior facilidade de acesso a mecanismos e instituições de financiamento; acesso a mercados e networking; processos de acompanhamento, avaliação e orientação sobre diversos aspectos, tais como: administrativos, comerciais, econômicofinanceiros, jurídicos e tecnológicos (WONGLIMPIYARAT, 2014).

As incubadoras visam proporcionar a base e o suporte necessários às empresas que estão incubadas para que tenham condições de ingressar e se manterem no mercado, diminuindo as taxas de mortalidade evidenciadas pelas pesquisas do Serviço Brasileiro de Apoio às Micro e Pequenas Empresas - SEBRAE.

Nessa perspectiva, contribuem para o desenvolvimento socioeconômico, uma vez que potencializam o surgimento de unidades produtivas, geram riquezas e postos de trabalho. Além disso, a incubadora favorece a criação de parcerias e redes de relacionamento que buscam promover um ambiente propício ao empreendedorismo e à inovação, fatores fundamentais para a viabilização de novos negócios.

\subsubsection{Incubadora Tecnológica Natal-Central do IFRN}

A primeira incubadora de empresas do Instituto Federal de Educação, Ciência e Tecnologia do Rio Grande do Norte, à época Escola Técnica Federal do Rio Grande do Norte - ETFERN surgiu em 1998, por meio do antigo Núcleo de Incubação Tecnológica (NIT), criado através da Resolução $n^{\circ}$ 02/1994-CTC.

O projeto nasceu com a missão de apoiar o surgimento de empresas de serviços e/ou produtos com inovação tecnológica, como forma de promover a geração de empregos e o desenvolvimento de pesquisas tecnológicas dentro das áreas de automação industrial, eletrônica, eletrotécnica, eletromecânica, construção civil, conservação de energia, controle de poluentes, desenvolvimento de aplicativos de softwares, energias renováveis, geologia e mineração, 
metalurgia, tecnologia de alimentos, tecnologia de saneamento e tratamento de água, tratamento de resíduos industriais e áreas afins (CTC-ETFRN, 1994).

Em 2011, a fim de atender à "Lei de Inovação", Lei no 10.973, de 2 de dezembro de 2004, que estabelece em seu Art. 16 que as Instituições de Ciência e Tecnologia - ICT deverão dispor de núcleo de inovação tecnológica, próprio ou em associação com outras ICT, com a finalidade de gerir uma política de inovação, bem como o Decreto $n$ 0 5.563/2005 que regulamenta a referida lei, foi criado o Núcleo de Inovação Tecnológica, órgão vinculado à Pró-Reitoria de Pesquisa e Inovação, responsável pelo Programa de Multincubação Tecnológica (MIT) do Instituto. Desde então, o programa tem avançado na difusão da cultura empreendedora de inovação e tecnologia, por meio do desenvolvimento de novos empreendimentos.

A Incubadora Tecnológica Natal-Central (ITNC) é a incubadora âncora do Instituto, instalada no Campus Natal-Central, tem seu público-alvo voltado para os estudantes dos campi, alunos egressos, pesquisadores e desenvolvedores de novas tecnologias e empreendedores em geral. A ITNC tem como objetivo fornecer suporte ao surgimento de empresas de base tecnológica, principalmente, com foco nas áreas de atuação do Instituto, e, já graduou 17 empresas (ITNC, 2015). Conforme evidenciam Santos et al (2005), as estratégias de valorizar atitudes de integração a fim de elevar a quantidade de empreendedores por oportunidades desenvolvendo a capacidade para executar negócios a partir de projetos é a vocação que a instituição vislumbra e pratica.

Atualmente, a ITNC inicia novas ações e atividades voltadas à implantação do modelo Cerne da Anprotec. Esse modelo prevê o aperfeiçoamento contínuo de diversos processos-chave, em especial, nas dimensões do desenvolvimento pessoal do empreendedor, desenvolvimento tecnológico de produtos e processos, aperfeiçoamento das estratégias de gestão empresarial, comercial, financeira e de marketing. O Cerne (Centro de Referência para Apoio a Novos Empreendimentos) é uma plataforma de soluções que visa ampliar a capacidade da incubadora em gerar, sistematicamente, empreendimentos inovadores bem-sucedidos. Para isso, determina boas práticas a serem adotadas em processos-chave presentes nos seguintes eixos: empreendedor, tecnologia, mercado, capital e gestão (ANPROTEC, 2015).

Os serviços oferecidos aos empreendedores iniciam antes da incubação e incluem a sensibilização para o empreendedorismo, a prospecção de novas empresas e a qualificação dos novos empreendedores. Durante a incubação, os empreendedores contam com assessoria e consultoria tecnológica, financeira, de mercado e de gestão, bem como acompanhamento e avaliação dessas áreas.

Os empreendedores também dispõem da infraestrutura integral do instituto, como: bibliotecas, auditórios, laboratórios, sala de reunião, sala de treinamento, sala de coordenação, estacionamento e restaurante.

Em parceria com o Sebrae e com a Fundação de Apoio à Educação e ao Desenvolvimento Tecnológico do Rio Grande do Norte (Funcern), ainda oferece às empresas alguns serviços especializados como: assessoria na elaboração dos planos de negócios, consultorias especializadas, prospecção tecnológica, participação em feiras, exposições, visitas técnicas, missões comerciais e cursos de qualificação e aperfeiçoamento.

As empresas que participam do projeto têm a oportunidade de estudar a viabilidade do seu negócio, uma vez que são inseridas em um roteiro básico de um contexto empresarial, no qual 
suas ideias ganham forma, estrutura e dimensão por meio da transferência de conhecimento e informações estratégicas para que sejam criadas e orientadas por um planejamento efetivo e consistente que lhes proporcione autonomia e autossustentação. Seguindo esse parâmetro, de acordo com Silva et al (2013), a incubadora visa à ampliação da competitividade de micro e pequenos empreendedores locais, com ênfase no desenvolvimento regional.

\subsection{Capacidade de Inovação}

Para se tornar uma organização bem-sucedida, considerando a complexidade do ambiente e necessidade de vantagem competitiva, a empresa precisa desenvolver e implementar novas ideias, uma vez que o seu desempenho depende da sua capacidade de inovação. Dada a relevância que a inovação tem para o sucesso da empresa, é fundamental identificar e analisar sua capacidade de inovação.

A capacidade de inovação é a habilidade empresarial de extrair conhecimentos das atividades rotineiras e das atividades inovadoras. Requer visão estratégica, inteligência organizacional, gestão de criatividade e ideias, estrutura organizacional, cultura e clima organizacional e gestão da tecnologia. É o potencial interno de uma empresa, na identificação de novas ideias, mercados e oportunidades (LAWSON; SAMSON, 2001).

Segundo Molina-Palma (2004), a capacidade de inovação está determinada pelas interrelações da cultura organizacional e relações interorganizacionais. Também compreende a capacidade de inovação como atividade realizada por pessoas que corresponde à cultura organizacional das instituições elencados por gestão do conhecimento, estilo gerencial e liderança, recursos, estrutura organizacional, estratégia corporativa e tecnologia.

Quadro 2: Determinantes e Resultantes da Capacidade de Inovação

\begin{tabular}{|c|c|c|}
\hline Fator & Domínio & Referências \\
\hline $\begin{array}{l}\text { Liderança } \\
\text { transformadora }\end{array}$ & $\begin{array}{l}\text { Aquela que torna seus seguidores mais conscientes } \\
\text { da importância e do valor do trabalho; ativa suas } \\
\text { necessidades de ordem superior; e os induz a } \\
\text { transcender } \quad \text { seus } \\
\text { pessoais em prol da organização. }\end{array}$ & $\begin{array}{l}\text { Avolio et al. (1999); } \\
\text { Podsakoff, MacKenzie, } \\
\text { Moorman e Fetter (1990). }\end{array}$ \\
\hline $\begin{array}{l}\text { Intenção estratégica } \\
\text { de inovar }\end{array}$ & $\begin{array}{l}\text { Grau que a empresa está disposta a assumir riscos } \\
\text { para favorecer a mudança, o desenvolvimento } \\
\text { tecnológico e a inovação, e a competir } \\
\text { agressivamente a fim de obter uma vantagem } \\
\text { competitiva para sua empresa. }\end{array}$ & $\begin{array}{l}\text { Covin e Slevin (1989); } \\
\text { Miller (1983). }\end{array}$ \\
\hline $\begin{array}{l}\text { Gestão de pessoas } \\
\text { para inovação }\end{array}$ & $\begin{array}{l}\text { Orientação da gestão de pessoas para a inovação, } \\
\text { provendo a concessão de liberdade ou autonomia de } \\
\text { atuação aos empregados, estabelecendo metas } \\
\text { desafiadoras, permitindo que decidam como alcançá- } \\
\text { las e favorecendo a autorrealização e o } \\
\text { comprometimento com os objetivos da organização. }\end{array}$ & $\begin{array}{l}\text { Amabile (1998); Amabile, } \\
\text { Schatzel, Moneta e } \\
\text { Kramer. } \\
\text { (2004); Cummings e } \\
\text { Oldham (1997); Mumford } \\
\text { etal. (2002); Shalley e } \\
\text { Gilson (2004). }\end{array}$ \\
\hline $\begin{array}{l}\text { Conhecimento do } \\
\text { cliente e do mercado }\end{array}$ & $\begin{array}{l}\text { Habilidade para detectar os eventos, necessidades, } \\
\text { expectativas, mudanças significativas e tendências do } \\
\text { cliente e do mercado. Perceber as mudanças do } \\
\text { mercado na frente dos seus competidores fornece } \\
\text { vantagem competitiva à empresa. }\end{array}$ & $\begin{array}{l}\text { Day (1994); Kohli e } \\
\text { Jaworski (1990). }\end{array}$ \\
\hline $\begin{array}{l}\text { Gestão estratégica } \\
\text { da tecnologia }\end{array}$ & $\begin{array}{l}\text { Gestão do processo de criação e desenvolvimento de } \\
\text { tecnologias, visando à criação de valor. O processo de }\end{array}$ & $\begin{array}{l}\text { A. R. Fusfeld (2004); H. I. } \\
\text { Fusfeld (1995); Phaal, }\end{array}$ \\
\hline
\end{tabular}




\begin{tabular}{l|l|l} 
& $\begin{array}{l}\text { gestão tecnológica compreende cinco etapas: } \\
\text { identificação, seleção, aquisição, explotação e } \\
\text { proteção. }\end{array}$ & $\begin{array}{l}\text { Farrukh e Probert (2001); } \\
\text { Gregory (1995). }\end{array}$ \\
\hline \multirow{5}{*}{$\begin{array}{l}\text { Organicidade da } \\
\text { estrutura organizacional que a estrutura é caracterizada pela } \\
\text { concessão de autonomia, controles flexíveis, } \\
\text { comunicação horizontal desimpedida, valorização do } \\
\text { conhecimento e da experiência e informalidade nas } \\
\text { relações pessoais. Estruturas ditas orgânicas } \\
\text { permitem resposta mais rápida às mudanças no } \\
\text { ambiente externo do que as denominadas } \\
\text { mecanicistas. }\end{array}$} & $\begin{array}{l}\text { Burns e Stalker (1961); } \\
\text { Khandwalla (1977). }\end{array}$ \\
\hline \multirow{5}{*}{ Gestão de projetos } & $\begin{array}{l}\text { Planejamento, provisão dos recursos, execução e } \\
\text { controle do processo de inovação. Inclui cuidadosa } \\
\text { avaliação dos projetos, análise e planejamento } \\
\text { visando, principalmente, ganhar compreensão, } \\
\text { compromisso e apoio tanto corporativo quanto do } \\
\text { pessoal que estará envolvido no projeto. }\end{array}$ & $\begin{array}{l}\text { Cooper e Klein Schmidt } \\
\text { (2007); Rothwell (1994). }\end{array}$ \\
\hline $\begin{array}{l}\text { Organizações inovadoras são aquelas que exibem } \\
\text { comportamento inovador consistente ao longo do } \\
\text { tempo. }\end{array}$ & $\begin{array}{l}\text { Meyer-Krahmer (1984); } \\
\text { Ritter e Gemünden (2004); } \\
\text { Prajogo e Ahmed (2006); } \\
\text { Subramanian e Nilakanta } \\
\text { (1996). }\end{array}$ \\
\hline
\end{tabular}

Fonte: Valladares et al. (2014).

Lemos (1999), por sua vez, disse ser relevante a aquisição de novas capacitações e conhecimentos, intensificar a capacidade e transformar esse aprendizado em fator de competitividade. Esse modelo limita a capacidade de inovação deixando de incluir o relacionamento de clientes como fator pertinente. O modelo de inovação de Valladares (2014) enfatiza a função cognitiva, a liderança, o modelo de gestão e o processo de inovação imerso na cultura organizacional.

À luz da literatura abordada por Ukko e Saunila (2012), a capacidade de inovação pode ser definida sob três elementos: 1) potencial de inovação: consiste em fatores que afetam o estado atual da capacidade de produzir inovações; 2 ) processos de inovação: são os sistemas e atividades que ajudam as organizações a utilizarem o seu potencial de inovação e, portanto, permitem inovações; e 3) resultados das atividades de inovação: são os novos produtos ou inovações em serviços resultantes dos processos.

Valladares et al (2014), após revisão bibliográfica sobre o desenvolvimento da capacidade de inovação nas organizações, constatou que há uma convergência em diversas correntes de estudos que permitiu identificar os principais fatores determinantes e resultantes da capacidade de inovação, sintetizadas no quadro 2 .

O quadro constitui um modelo teórico pelo qual se identifica um conjunto de fatores decisivos para o desempenho em inovação da organização. Valladares (2012) aponta como principais fatores determinantes da inovação: liderança transformadora, intenção estratégica de inovar, gestão de pessoas para inovação, conhecimento do cliente e do mercado, gestão estratégica da tecnologia, organicidade da estrutura organizacional e gestão de projetos. $\mathrm{O}$ autor constata ainda que o desempenho em inovação abrange a inovação de processos ou de produtos, ou seja, é resultante da capacidade de inovação. 


\section{METODOLOGIA}

O presente artigo propõe uma pesquisa qualitativa descritiva, uma vez que pretende identificar e compreender a percepção da equipe da Incubadora Tecnológica Natal-Central do IFRN quanto à capacidade de inovação das empresas nela incubadas. Portanto, para atender os objetivos do trabalho, foi realizada pesquisa bibliográfica e documental, bem como um estudo de caso. Quanto à coleta de dados optou-se por conduzir entrevistas semiestruturadas, cujos dados foram tratados segundo as práticas de análise de conteúdo.

A pesquisa qualitativa foi julgada mais apropriada tendo em vista a preocupação com os aspectos da realidade das empresas incubadas, centrando-se na compreensão e explicação das relações sociais e no universo de significados, motivos, aspirações, crenças, valores e atitudes que envolvem a capacidade de inovação dessas empresas (GERHARDT; SILVEIRA, 2009).

A pesquisa descritiva baseia-se na descrição dos fatos e fenômenos da realidade (MALHOTRA, 2005). Neste trabalho, preocupou-se em apresentar as principais abordagens sobre inovação, caracterizar as incubadoras e descrever a percepção da equipe da ITNC sobre o tema investigado.

Para obter as informações necessárias foram conduzidas quatro entrevistas semiestruturadas em profundidade. Dessa forma, permitiu-se explorar os pontos de vista dos atores sociais inseridos no contexto de investigação, permitindo compreender os fenômenos organizacionais e ampliar o conhecimento nesse campo de investigação (OLIVEIRA et al., 2012).

As entrevistas foram organizadas sob um roteiro com 14 questões-chaves elaboradas a partir dos constructos determinantes e dos constructos resultantes da capacidade de inovação levantados por Valladares (2012). No decorrer das entrevistas, realizadas com a equipe da ITNC, foi discutido como eles percebem os aspectos internos de liderança transformadora, organicidade da estrutura organizacional, intenção estratégica de inovar e o conhecimento sobre o cliente e o mercado das empresas incubadas na ITNC. E ainda, como percebem a gestão estratégica de tecnologia, gestão de projetos, gestão de pessoas para inovação e como esses elementos afetam a sistemática de inovação de produtos e processos.

Com o objetivo de interpretar o material coletado, foi empregada a técnica denominada análise de conteúdo a qual, segundo Bardin (2009), consiste em um conjunto de técnicas de análise das comunicações que visam obter, por meio de procedimentos sistemáticos e descrição do conteúdo das mensagens obtidas, indicadores que permitam a inferência de conhecimentos relativos às condições de produção e recepção dessas mensagens.

Do ponto de vista operacional, foi feita uma leitura flutuante da transcrição das entrevistas e partir daí, pode-se categorizar os fatores determinantes e resultantes da capacidade de inovação identificadas nas falas. Posteriormente, como recomenda Bardin (2009), o material foi explorado e organizado em categorias para, em seguida, ser tratado e interpretado.

\section{RESULTADOS}

A Incubadora Tecnológica Natal-Central (ITNC) é a incubadora matriz do Instituto Federal de Educação, Ciência e Tecnologia do Rio Grande do Norte, instalada no Campus Natal-Central. Em 
seus 17 anos de funcionamento já graduou 17 empresas e hoje abriga 12 empresas que contam com uma infraestrutura física exclusiva com mais de $700 \mathrm{~m}^{2}$.

A ITNC tem como objetivo prospectar, capacitar e fortalecer empresas ou empreendimentos que apresentem produtos, processos ou serviços inovadores, por meio da promoção de serviços especializados de apoio técnico e operacional. Dessa forma, a incubadora atua no sentido de minimizar o risco de falência das empresas nos primeiros anos de desenvolvimento dos seus produtos e serviços e, em especial, durante a fase inicial de inserção no mercado concorrencial (ITNC, 2015).

As empresas incubadas atuam no desenvolvimento de novos produtos, processos e serviços nos quais aplicam conhecimentos científicos na geração da inovação em diversas plataformas e tecnologias. Trabalham na criação de aplicativos, lojas virtuais, softwares, sites, interfaces gráficas, sistemas web. Há também empresas focadas no desenvolvimento de produtos e soluções inovadoras de base tecnológica para os mercados de saneamento, uso sustentável dos recursos hídricos; serviços de licenciamento, gerenciamento, operação e monitoramento do setor ambiental; geoprocessamento, banco de dados para exploração mineral, geofísica, sensoriamento remoto, topografia e geomarketing; gestão hospitalar; marketing digital; inteligência competitiva e dispositivos de localização.

Foram aplicadas entrevistas semiestruturadas em que os inquiridos foram pessoas da equipe da ITNC, por meio das quais buscou-se avaliar a capacidade de inovação das empresas incubadas na perspectiva da incubadora. Traçaram-se, então, os fatores que deveriam ser avaliados: liderança transformadora, intenção estratégica de inovar, gestão de pessoas para inovação, conhecimento do cliente e do mercado, gestão estratégica da tecnologia, organicidade da estrutura organizacional, gestão de projetos e desempenho em inovação.

Nesse contexto, observou-se que a equipe, formada por quatro pessoas, aqui identificada como entrevistado $A$, entrevistado $B$, entrevistado $C$ e entrevistado $D$, considera a ITNC de suma importância para o desenvolvimento socioeconômico do estado, uma vez que fomenta o empreendedorismo, proporciona inovação ao mercado potiguar mediante a inserção de novos produtos e serviços, ajuda as empresas a desenvolverem suas habilidades e potencialidades, aumentando, assim, suas chances de sobrevivência no mercado.

Para ingressar na ITNC, as empresas passam por um processo seletivo pelo qual o empreendimento a ser incubado deverá ter o modelo de negócio aprovado conforme critérios estabelecidos no edital. Durante esse processo, os principais elementos analisados são: capacidade de gestão do negócio, marketing, tecnologia, capital e perfil empreendedor. Além disso, também é avaliado o quão a ideia é inovadora e se é capaz de gerar um produto ou serviço comercializável.

Com base em sua atuação, a equipe da ITNC concorda que a incubadora exerce influência sobre a capacidade de inovação das empresas incubadas. O entrevistado A declarou que as empresas já trazem um produto ou serviço inovador, porém, a ITNC é fundamental na lapidação e no aperfeiçoamento dessas criações até que cheguem ao mercado. $O$ entrevistado $B$ acrescentou que as empresas são estimuladas continuamente a manterem inovação em seus produtos $\mathrm{e}$ serviços. $O$ entrevistado $C$, por sua vez, destacou a multidisciplinaridade da equipe de suporte, a promoção de capacitações de acordo com a necessidade do empreendedor, a participação em eventos e o ambiente de inovação e cooperação no qual as incubadas estão inseridas. Sob este 
último aspecto, o entrevistado $D$ afirmou, ainda, que o ambiente dinâmico e interativo, dotado de práticas inovadoras de negócios, fortalece a capacidade de inovação dessas empresas.

Sob os aspectos da capacidade de inovação das empresas incubadas na ITNC, foram identificadas nas entrevistas os constructos determinantes e os constructos resultantes da capacidade de inovação abordados por Valladares et al (2014), conforme o quadro a seguir:

Quadro 3: Fatores determinantes e fatores resultantes da Capacidade de Inovação identificados

\begin{tabular}{|l|c|c|c|c|}
\hline Fatores & Entrevistado & Entrevistado & Entrevistado & Entrevistado \\
D & B & $\checkmark$ & \\
\hline Intenção estratégica de inovar & & $\checkmark$ & $\checkmark$ & \\
\hline Liderança transformadora & $\checkmark$ & $\checkmark$ & $\checkmark$ & $\checkmark$ \\
\hline Organicidade da estrutura organizacional & $\checkmark$ & $\checkmark$ & $\checkmark$ & $\checkmark$ \\
\hline Gestão de pessoas para inovação & & $\checkmark$ & $\checkmark$ & $\checkmark$ \\
\hline Conhecimento do cliente e do mercado & & $\checkmark$ & $\checkmark$ & $\checkmark$ \\
\hline Gestão estratégica da tecnologia & $\checkmark$ & $\checkmark$ & & \\
\hline Gestão de projetos & & $\checkmark$ & $\checkmark$ & $\checkmark$ \\
\hline Desempenho em inovação & $\checkmark$ & $\checkmark$ & & $\checkmark$ \\
\hline
\end{tabular}

Fonte: Resultados da pesquisa (2015).

Ao questionar sobre a intenção estratégica de inovação das empresas incubadas e sobre sua eficiência, constatou-se que estas chegam a ITNC sem preparo gerencial, no máximo utilizam, segundo o entrevistado $B$, um roteiro de planejamento do produto ou serviço que estão desenvolvendo. Durante o processo de incubação, conforme relataram os entrevistados $C$ e $D$, as empresas passam por uma oficina de modelagem de negócio, na qual têm a oportunidade de desenvolver o modelo de negócios da empresa de uma forma simples e visual através da metodologia Business Model Canvas $^{1}$ - ferramenta de gestão estratégica e empresarial que permite planejar e visualizar as principais funções de um negócio e suas relações e ainda fornece uma visão holística e flexível do modelo de negócios, auxiliando os empreendedores nos processos de criação, de diferenciação e de inovação dos produtos e serviços.

Outra ferramenta utilizada, de acordo com o entrevistado C, é a elaboração do Plano de Ação, no qual os objetivos e metas são definidos e há por parte da ITNC um acompanhamento da evolução e do cumprimento do que foi planejado. De forma geral, os entrevistados concordam que essas ferramentas atendem os empreendedores de forma positiva. No entanto, salientam que, tratando-se de inovação, nada é estático e diante da dinamicidade do ambiente e da forma como ocorrem as mudanças, tais ferramentas podem tornar-se insuficientes e outros modelos podem surgir.

Em relação à liderança transformadora, a maioria identificou a capacidade de gerenciamento das pessoas no sentido de gerar resultados, influenciando, dessa forma, no desempenho da empresa. Para o entrevistado A, o perfil de liderança é essencial para gerir o negócio. $O$ entrevistado $C$ pontuou que cada empresa apresenta suas particularidades, algumas têm uma equipe, outras têm apenas um membro (o proprietário), contudo, completou $C$, em ambos os casos existe a figura do líder. Entretanto, o entrevistado $D$ acredita que os empreendedores incubados são bons desenvolvedores dos seus produtos, porém, nem todos sabem como exercer um papel diferente, como a liderança, no empreendimento. Para estes

${ }^{1}$ Disponível em: <http://www.businessmodelgeneration.com/canvas/bmc e <http://www.sebrae.com.br/sites/Por talSebrae/bis/Quadro-de-modelo-de-neg\%C3\%B3cios:-para-criar,-recriar-e-inovar>. 
últimos, o entrevistado $B$ frisou que os que não possuem o perfil de liderança procuram se capacitar.

Quando perguntado sobre a organicidade da estrutura organizacional das empresas abrigadas na ITNC, os entrevistados foram unânimes ao afirmarem que as empresas são flexíveis e adaptáveis a mudanças circunstanciais do mercado e estruturam o seu planejamento de acordo com as necessidades do negócio. Observou-se também que a comunicação é horizontal, acontece naturalmente, sem formalidades e barreiras assim como os controles gerenciais.

Ainda sobre a organicidade das empresas, foi consultado como a equipe da ITNC percebe a cultura da inovação nas empresas incubadas. Todos os entrevistados responderam que existe uma cultura de inovação nas empresas, presente desde o seu recrutamento. Os entrevistados A e B acrescentaram que essas empresas estão sempre participando de cursos, se atualizando, compartilhando informações, buscando melhorias para os seus produtos ou serviços e firmando parcerias com outras instituições. Isso demonstra que seus hábitos, comportamentos, crenças e valores são voltados para inovação.

Referente à gestão de pessoas para inovação, as empresas incubadas quase na sua totalidade têm poucas pessoas na equipe ou contam apenas com o proprietário. No que se refere a um modelo de gestão, as empresas não estabeleceram ainda, metas para a equipe, pois ainda estão iniciando. Contudo, observou-se nas falas que existem pessoas aplicadas, integradas, motivadas e comprometidas, atitudes pontuais que influenciam no desempenho das empresas.

Dando prosseguimento, notou-se que as empresas estão atentas ao cliente e ao mercado. Segundo os entrevistados, elas utilizam ferramentas de mapeamento de mercado, como o software próprio baseado Business Model Canvas, para buscar o máximo de conhecimento do mercado e seus concorrentes. Ademais, o processo de incubação envolve a validação do produto ou serviço antes de iniciar a comercialização dos mesmos, o que permite perceber melhorias e minimizar os riscos. Nessa etapa é preciso conhecer o cliente que se quer trabalhar.

Quanto à gestão estratégica de tecnologia utilizada, em que o processo de gestão se processa na criação e desenvolvimento de tecnologias visando à criação de valor, a equipe entrevistada concorda que essa gestão é importante para que as empresas desenvolvam produto inovador que tenham impacto no mercado. $O$ entrevistado $A$ salientou que essa questão está ligada à inovação, cujas novas tecnologias permeiam praticamente todos os produtos. Para ele, empreender tecnologias inovadoras desenvolvidas em um ambiente de base tecnológica com infraestrutura diferenciada é um diferencial no desenvolvimento de novas tecnologias.

Em relação à gestão de projetos é importante salientar a importância do planejamento, provisão dos recursos, execução, controle do processo que inclui uma avaliação dos projetos, visando compromisso e apoio de toda equipe que está envolvida no projeto. Os entrevistados percebem que as empresas utilizam alguns softwares onde fazem o acompanhamento de seus projetos, porém ainda não despertaram para a importância desse fator determinante para a capacidade de inovação.

Quanto aos obstáculos, na visão dos entrevistados as empresas incubadas enfrentam os mesmos obstáculos que as empresas de todo o Brasil. O fato delas receberem todo o apoio das incubadoras, com infraestrutura, capacitações, consultorias são relevantes para o desenvolvimento do negócio. No entanto, falta investimento tanto de investidores, quanto da 
própria incubadora, para manter a estrutura e o fluxo de negócios. $O$ entrevistado A esclarece que um grande problema enfrentado é a falta de investidores que estejam dispostos a conhecer os produtos que estão sendo desenvolvidos e investir nesses produtos. Ele complementa que, se houvesse mais pessoas preocupadas com a questão da inovação, as empresas incubadas teriam mais possibilidades de crescimento e sucesso garantido.

Ainda segundo o entrevistado $A$, há dificuldades, por exemplo, de enviar as empresas para participar de eventos fora do Brasil, que possibilitam conhecer a realidade em outros países e adquirir experiências novas que certamente agregarão valor à empresa. O Sebrae tem financiado projetos, mas ainda é pouco, pois a necessidade é muito maior, e a própria instituição ainda não dá o apoio financeiro necessário para que muitas empresas que ainda não estão gerando negócio, possam se desenvolver e trocar experiências com outras do Brasil e do mundo. Afinal, ele conclui, é o nome da instituição que está sendo levado para os diversos lugares em que as empresas têm passado.

\section{CONCLUSÃO}

O presente estudo teve a finalidade de compreender a capacidade de inovação das empresas incubadas na perspectiva da equipe da ITNC do IFRN. Para atingir esse objetivo foram realizadas entrevistas com a equipe da ITNC, cujos resultados foram comparados aos fatores determinantes e relacionados aos fatores resultantes da capacidade de inovação levantados durante a pesquisa.

A capacidade de inovação está fundamentada sob a avaliação dos aspectos de liderança transformadora, organicidade da estrutura organizacional, intenção estratégica de inovar, conhecimento sobre o cliente e o mercado, gestão estratégica de tecnologia, gestão de projetos, gestão de pessoas para inovação e como parâmetros interferem na inovação de produtos, serviços ou processos das empresas.

A partir dos resultados das entrevistas, pode-se identificar, do ponto de vista da equipe da ITNC, nas empresas incubadas, a presença dos oito fatores que constituem a capacidade de inovação, sendo a intenção estratégica de inovar e a gestão de projetos percebidos de maneira mais sutil e a organicidade da estrutura organizacional e a gestão estratégica da tecnologia notadas como predominantes no dia a dia dessas empresas.

Endente-se que os resultados encontrados apontam que as empresas incubadas demonstram intenção estratégica de inovar. Isso se justifica quando se considera o fato de procurarem uma incubadora com o intuito de se colocar no mercado. Tal atitude as coloca em um processo de planejamento estratégico, mesmo que não tenham preparo gerencial ou um documento formal para isso. E tão logo ingressem na ITNC e mostram-se comprometidas com os objetivos e metas organizacionais.

Os achados também revelam que existe nas empresas incubadas a ação de liderança transformadora empenhada em motivar as pessoas em um ambiente de confiança e colaboração, gerando resultados satisfatórios. Além disso, notou-se, ainda, que as empresas firmam-se sobre estruturas organizacionais flexíveis, permitindo um maior grau de autonomia entre os membros, prevalecendo o espírito de equipe na tomada de decisões e na obtenção dos resultados. 
Evidencia-se na compreensão da equipe da incubadora que, apesar das limitações de funcionários, as empresas incubadas desenvolvem a gestão de pessoas para inovação quando compartilham a visão geral do negócio, da liderança, da flexibilidade e da autonomia em um clima organizacional que favorece a criatividade e o apoio integral das equipes aos objetivos das empresas.

Outro fator identificado na pesquisa é o conhecimento do cliente e do mercado que existe nas incubadas. Percebeu-se que elas buscam, desde a sua concepção, informações sobre as necessidades e preferências dos clientes e associam a ideia do negócio às oportunidades e tendências do mercado, vislumbrando a validação e comercialização do seu produto/serviço.

A partir da observação da equipe, as empresas, além de desenvolverem novas tecnologias, preocupam-se com os subsídios, a análise e o acompanhamento de seus projetos, assegurando um gerenciamento eficaz quanto ao crescimento e sustentação da empresa.

De modo similar, há uma análise positiva da equipe da ITNC quanto à gestão de projetos, quando se observa que as empresas incubadas dispõem de planejamento e controle das suas criações. O processo inicia-se na seleção das empresas para incubação, passa pela oficina de modelagem de negócio (Business Model Canvas), aprimoramento do produto/serviço, elaboração do plano de negócios e validação para, enfim, ser comercializado. Cabe lembrar que há nas empresas compreensão, compromisso e apoio das equipes envolvidas nos projetos.

Diante do exposto, os dados comprovam que, segundo a equipe da ITNC, as empresas nela incubadas exibem o comportamento inovador replicado na teoria ora apresentada. Dotadas de novas ideias, criatividade, planos e estratégias, inovação em produtos, serviços e processos, suas competências compreendem os principais fatores determinantes da capacidade de inovação e demonstram seu relevante desempenho em inovação.

Com os resultados alcançados, almeja-se contribuir na disseminação do conhecimento sobre capacidade de inovação nas empresas, incentivando, inclusive, novos estudos sobre inovação. Sugere-se, dessa forma, para futuras pesquisas, mensurar a capacidade de inovação de micro e pequenas empresas, ou de empresas incubadas em outras instituições.

\section{REFERÊNCIAS}

1. AL-MUBARAKI, Hanadi Mubarak; BUSLER, Michael. Incubator successes: Lessons learned from successful incubators towards the twenty-first century. World Journal of Science, Technology and Sustainable Development, v. 11, Iss: 1, pp. 44 - 52, 2014.

2. ARAÚJO, Victor Ranieri Bomfim Sampaio de. et al. Avaliação de Empresas: um estudo em uma empresa incubada no Estado de Alagoas. In: 30a Conferência Mundial IASP de Parques Científicos e Tecnológicos e XXIII Seminário Nacional de Parques Tecnológicos e Incubadoras de Empresas, APROTEC, Recife, 2013.

3. ASSOCIAÇÃO NACIONAL DE ENTIDADES PROMOTORAS DE EMPREENDIMENTOS INOVADORES. Disponível em: <http://anprotec.org.br/site/menu/incubadoras-e-parques/perguntasfrequentes/> Acesso em: 31 ago. 2015.

4. ASSOCIAÇÃO NACIONAL DE ENTIDADES PROMOTORAS DE EMPREENDIMENTOS INOVADORES; SERVIÇO BRASILEIRO DE APOIO ÀS MICRO E PEQUENAS EMPRESAS. Planejamento $\mathrm{e}$ 
implementação de incubadoras de empresas. Brasília: Anprotec/Sebrae, 2002.

5. ASSOCIAÇÃO NACIONAL DE ENTIDADES PROMOTORAS DE EMPREENDIMENTOS INOVADORES. Estudo, análise e proposições sobre as Incubadoras de Empresas no Brasil. Relatório técnico 2012. Brasília: ANPROTEC, 2012.

6. BARDIN, Laurence. Análise de conteúdo. 4 ed. Lisboa: Edições 70, LDA. 2009.

7. BASSAN, Dilani Silveira. Alguns aspectos teóricos do desenvolvimento com o olhar na região do Vale do Paranhana. RDE - Revista de Desenvolvimento Econômico, v. 16, n. 29, 2014.

8. BRASIL. Lei no 10.973 , de 2 de dezembro de 2004. Dispõe sobre incentivos à inovação e à pesquisa científica e tecnológica no ambiente produtivo e dá outras providências. Disponível em: < http://www.planalto.gov.br/ccivil_03/_ato2004-2006/2004/lei/l10.973.htm>. Acesso em: 31 ago. 2015.

9. BRASIL. Decreto № 5.563, de 11 de outubro de 2005. Regulamenta a Lei no 10.973 , de 2 de dezembro de 2004, que dispõe sobre incentivos à inovação e à pesquisa científica e tecnológica no ambiente produtivo, e dá outras providências. Disponível em: < http://www.planalto.gov.br/ccivil_03/_ato2004-2006/2005/decreto/d5563.htm>. Acesso em: 31 ago. 2015.

10. CONSELHO TÉCNICO CONSULTIVO DA ESCOLA TÉCNICA FEDERAL DO RIO GRANDE DO NORTE. Aprova Estatuto da Incubadora desta Escola Técnica Federal do Rio Grande do Norte. Resolução n. 2, de 15 ago. 1994.

11. CHURCHILL, N.C.; MUZYKA, D.F. High performance entrepreneurial teams, In Frontiers of Entrepreneurship Research, p. 503, 1995.

12. DE FELICE, A. Measuring the social capabilities and the implication on innovation. Journal of Economic Studies. 41, 6, 907-928, nov. 2014. ISSN: 01443585.

13. ENGEL, Kai; DIRLEA. Violetka; DYER, Stephen; GRAFF, Jochen.Best innovators develop a point of view on the future and a roadmap on how to get there. Strategy \& Leadership, v. 43, Iss 2 , pp. $15-22,2015$.

14. GERHARDT, Tatiana Engel; SILVEIRA, Denise Tolfo. Métodos de pesquisa. Porto Alegre: Editora da UFRGS, 2009. Disponível em: <http://www.ufrgs.br/cursopgdr/downloadsSerie/derad005 .pdf>. Acesso em: 20 set. 2015.

15. HASHIMOTO, Marcos. Espírito empreendedor nas organizações: aumentando a competitividade através do intra-empreendedorismo. São Paulo: Saraiva, 2006.

16. HENDERSON, Rebecca M.; CLARK, Kim B. Architectural Innovation: The Reconfiguration of Existing Product Technologies and the Failure of Established Firms. Administrative Science Quarterly, v. 35, n. 1, Special Issue: Technology, Organizations, and Innovation, pp. 9-30, 1990.

17. INCUBADORA TECNOLÓGICA NATAL-CENTRAL - Disponível em: <http://www2.ifrn.edu.br /itnc/novo/>Acesso em 28 ago. 2015.

18. LAWSON, Benn; SAMSON, Danny. Developing Innovation Capability in Organizations: A Dynamica Capabilities Approach. International Journal of Innovation Management. Vol. 5 no 3. P. 377-400. 2001.

19. LEMOS, Cristina. Inovação na Era do Conhecimento. In: LASTRES, H; ALBAGLI, S. (Org.). Informação e Globalização na Era do Conhecimento. Rio de Janeiro: Campus, 1999. 
20. MALHOTRA, Naresh K. et al. Introdução à pesquisa de marketing. São Paulo: Pearson Prentice Hall, 2005.

21. MOLINA-PALMA, Manuel Antonio. A capacidade de inovação como formadora de valor: análise dos vetores de valor em empresas brasileiras de biotecnologia. 2004. Tese (Doutorado em Administração) - Faculdade de Economia, Administração e Contabilidade, Universidade de São Paulo, São Paulo, 2004. Disponível em: <http://www.teses.usp.br/teses/disponiveis/12/ 12139/tde-10112004-212943/>. Acesso em: 2015-10-03.

22. MORAIS, Ednalva F. C. de. (Org) et al. Manual de Acompanhamento e Auto-avaliação de Incubadoras e Empresas Incubadas. Brasília: Editora Universidade de Brasília, 1997.

23. OCDE. Organização para a Cooperação e Desenvolvimento Econômico. Manual de Oslo: proposta de diretrizes para coleta e interpretação de dados sobre inovação tecnológica. 3. ed. Brasília: FINEP, 2005.

24. OLIVEIRA, Verônica Macário et al. Entrevistas "Em Profundidade" na Pesquisa Qualitativa em Administração: Pistas Teóricas e Metodológicas. In: Anais do SIMPOI 2012, São Paulo, 2012.

25. PAIVA JR., F. G. O empreendedor e sua identidade cultural: em busca do desenvolvimento local. In: Anais do XXVIII ENANPAD, Brasília, 2005.

26. QUINELLO, Robson; NICOLETTI, José Roberto. Inovação operacional. Rio de Janeiro: Brasport, 2009.

27. SANTOS, J.P; LEITE, J.Y.P; ARAÚJO, A.L.C. Empreendedorismo e Inovação Tecnológica - Uma Nova Metodologia. HOLOS, Natal, ano 21, pp. 36-46, 2005.

28. SEBRAE. Coleção Estudos e Pesquisas: Sobrevivência das Empresas no Brasil. Unidade de Gestão Estratégica: Brasília, 2013. Disponível em: <http://www.sebrae.com.br/Sebrae/ Portal\%20Sebrae/Anexos/Sobrevivencia_das_empresas_no_Brasil=2013.pdf > Acesso em: 25 ago. 2015.

29. SEBRAE. Modelo otimiza resultados de incubadoras do Rio Grande do Norte. Agência Sebrae de Notícias. http://www.agenciasebrae.com.br/sites/asn/uf/NA/Modelo-otimiza-resultadosde-incubadoras-do-Rio-Grande-do-Norte Acesso em 10 de set. 2015.

30. SCHUMPETER, Joseph Alois. Teoria do Desenvolvimento Econômico uma investigação sobre lucros, capital, crédito, juro e o ciclo econômico. 3. ed. São Paulo: Nova Cultura, 1997.

31. SILVA, R.L.S; D’AMORE, T.M; ARAÚJO, A.G; SILVEIRA, M.L.S.S. Incubadora e Gestão: Uma Percepção das Empresas Incubadas. HOLOS, Natal, ano 29, vol 3, pp. 27-37, 2013.

32. THEODORAKOPOULOS, Nicholas; KAKABADSE, Nada K.; MCGOWAN, Carmel. What matters in business incubation? A literature review and a suggestion for situated theorizing. Journal of Small Business and Enterprise Development, v. 21, Iss 4, pp. $602-622,2014$.

33. UKKO, Minna; SAUNILA, Juhani. A conceptual framework for the measurement of innovation capability and its effects. Baltic Journal of Management, v. 7, Iss 4, pp. 355-375, 2012.

34. VALLADARES, Paulo Sergio Duarte de Almeida. Capacidade de inovação: análise estrutural e o efeito moderador da organicidade da estrutura organizacional e da gestão de projetos. Tese (Doutorado em Administração de Empresas) - Escola de Administração de Empresas de São Paulo da Fundação Getúlio Vargas, São Paulo, 2012.

35. VALLADARES, Paulo Sergio Duarte de Almeida; VASCONCELLOS, Marcos Augusto; SERIO, Luiz 
Carlos Di. Capacidade de Inovação: Revisão Sistemática da Literatura. RAC, Rio de Janeiro, v. 18, n. 5, art. 3, pp. 598-626, Set/Out, 2014.

36. WONGLIMPIYARAT, Jarunee. Incubator policy to support entrepreneurial development, technology transfer and commercialization. World Journal of Entrepreneurship, Management and Sustainable Development, Vol. 10, Iss 4 pp. 334 - 351, 2014. 